\title{
Perforating branches from offending arteries in hemifacial spasm: anatomical correlation with vertebrobasilar configuration
}

\author{
Tetsuya Nagatani, Suguru Inao, Yoshio Suzuki, Jun Yoshida
}

\begin{abstract}
Objective-In microvascular decompression for hemifacial spasm, the perforating branches around the facial nerve root exit zone occasionally complicate facial nerve decompression. In this context, the vertebrobasilar configuration was retrospectively correlated with the perforating branches.
\end{abstract}

Methods-Based on vertebral angiography, magnetic resonance angiography, and three dimensional computed tomographic angiography, 69 patients were divided into three groups, according to the anatomy of the vertebrobasilar system. In patients with the type I configuration, the vertebral artery on the affected side was dominant and had a sigmoidal course. The type II patients had the basilar artery curving mainly towards the affected side. The type III patients showed the basilar artery either running straight or curving toward the unaffected side. The relation of the anatomical configuration of these vessels with the perforating branches around the facial nerve exit zone was investigated. Results-The posterior inferior cerebellar artery in type I patients $(n=33)$ and the anterior inferior cerebellar artery in type II $(n=5)$ and type III $(n=31)$ patients were the most common offending arteries. More than half of the type I patients $(n=20)$ showed no perforating branches around the facial nerve exit zone. However, the type II $(n=3)$ and III patients $(n=23)$ often showed one or more perforating branches around that region.

Conclusions-The configuration of the vertebrobasilar system has a significant correlation with the presence of perforating branches near the site of microvascular decompression. These perforating vessels are often responsible for the difficulty encountered in mobilising the offending artery during the procedure. (f Neurol Neurosurg Psychiatry 1999;67:73-77)

Keywords: hemifacial spasm; microvascular decompression; vertebrobasilar; perforating branch

Gardner and Sava are credited with the first published report of five patients with hemifacial spasm, who were successfully treated by placing a piece of prosthetic material between the internal auditory artery and the cochlear nerve ${ }^{1}$. Jannetta et $a l^{2}$ subsequently showed that the arterial compression of the facial nerve root exit zone was responsible for the facial spasm and standardised the technique of microvascular decompression. Although several therapeutic alternatives have been reported, ${ }^{3}$ microvascular decompression is widely accepted as the most effective and safe treatment for hemifacial spasm. Some potential difficulties, however, exist with this tecnique. Occasionally, surgeons have had to manipulate the small perforating branches around the root exit zone. This, however, can interfere with the mobilisation of the compressing vessels and the creation of space for the placement of the prosthetic materials without damage to the surrounding structures. Some authors have attempted to detect the offending vessels in radiological studies, ${ }^{4}$ but there are no reports available regarding the perforating branches around the root exit zone of the VIIth nerve. In this study, we have focused on the configuration of the vertebrobasilar system as seen on the preoperative imaging as a guide to the detection of the perforating branches encountered during microvascular decompression for hemifacial spasm.

\section{Patients and methods}

Seventy seven consecutive patients with hemifacial spasm were treated at our institution between July 1983 and April 1996. Sixty nine of these patients underwent microvascular decompression after angiography, magnetic resonance angiography (MRA), or three dimensional computed tomographic angiography (3-D CT angiography). The 69 operations for microvascular decompression, including three reoperations, were performed on 26 men and 43 women, with ages ranging from 24 to 72 years (mean age 50.6 years ). In 36 patients, facial spasm was found on the right side; in 33, it occurred on the left. No patient had bilateral manifestations. The hemifacial spasm was diagnosed by careful study of history and complemented by radiological imaging to rule out other aetiologies which may be responsible for spasm such as other vascular diseases or a mass lesion in the posterior fossa.

The operative procedure was similar to that previously described by Jannetta et $a l^{2}{ }^{2}$ We applied a piece of muscle as prosthesis until 1986. Since then, we have been using Tefloncotton, although other materials have been recommended as prostheses. ${ }^{5}{ }^{6}$

The relation between the microvascular structures including the perforating branches and the facial nerve was confirmed by reviewing operative notes or videos. The preoperative 


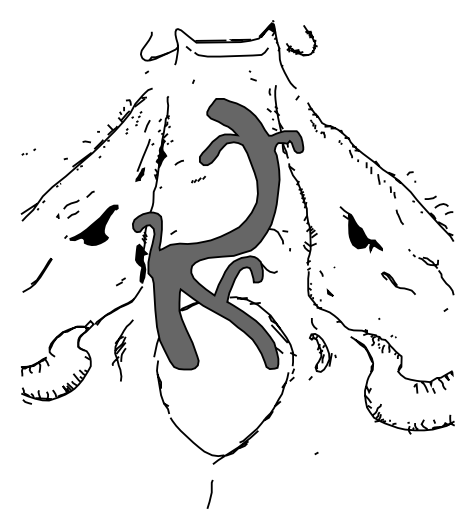

Type I

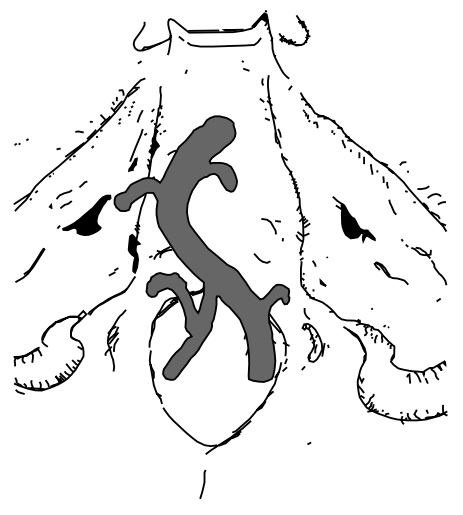

Type II

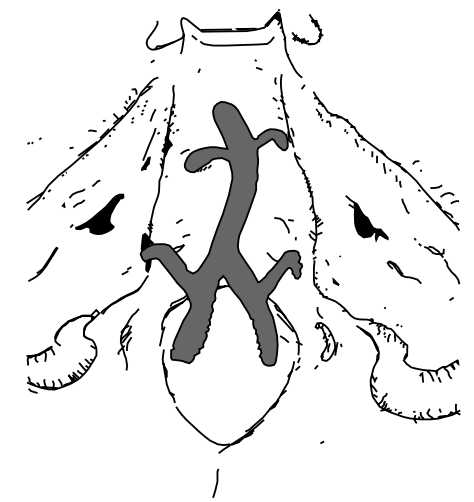

Type III

Figure 1 Schematic diagram of the three types of vertebrobasilar system. Type I: the vertebral artery ipsilateral to the spasm is dominant and has a sigmoidal course towards the affected side. Type II: the vertebral artery contralateral to the spasm is dominant and the basilar artery has a sigmoidal course away from the midline towards the affected side. Type III: the basilar artery runs relatively straight or is curving towards the unaffected side. The left side is the affected side.

Table 1 Distribution of the offending vessels in relation to the vertebrobasilar configuration

\begin{tabular}{lllll}
\hline \multirow{5}{*}{ Offending vessel } & \multicolumn{2}{l}{ Type of vertebrobasilar anatomy } & \\
\cline { 2 - 4 } & Type I (\%) & Type II (\%) & Type III (\%) & Total \\
\hline AICA & $6(18)$ & $5(100)$ & $25(81)$ & $36(52.1 \%)$ \\
PICA & $24(73)$ & 0 & $6(19)$ & $30(43.5 \%)$ \\
VA & $3(9)$ & 0 & 0 & $3(4.4 \%)$ \\
Total & 33 & 5 & 31 & 69 \\
\hline
\end{tabular}

$\mathrm{AICA}=$ anterior inferior cerebellar artery; $\mathrm{PICA}=$ posterior inferior cerebellar artery; $\mathrm{VA}=$ vertebral artery. Types I and III are significantly different ( $\mathrm{p}<0.01$, two way ANOVA).

duration of the symptoms ranged from 3 months to 16.7 years, with a mean duration of 73.7 months. We categorised the operative results at discharge as complete relief, partial relief, or no benefit. The imaging demonstrated that the vertebrobasilar anatomy followed one of three characteristic patterns. In the type I configuration, the vertebral artery ipsilateral to the spasm was dominant and sigmoidal in course. In type II, the vertebral artery contralateral to the spasm was dominant with the basilar artery deviated from the midline and curving mainly toward the affected side; In type III, the basilar artery remained near the midline or curved towards the unaffected side. The three types are shown in a schematic diagram in fig 1 . The compressing vessels and their perforating branches arising from the offending arteries were examined by reviewing the operative notes and divided into two groups according to the existence of the perforating branches around the compressing site. In each type of the vertebrobasilar configuration, the offending artery causing the hemifacial spasm and the presence or absence of the perforating vessels were also examined.

As a control group, we also included 62 patients (male: female ratio 25:37; age range 24-74 years; mean age 67.8 years) who had undergone either angiography, MR angio-

Table 2 Correlation between the various types of the vertebraobasilar system and the number of patients having perforating branches

\begin{tabular}{lllll}
\hline & Type I & Type II & Type III & Total \\
\hline Perforating branch (-) & 20 & 2 & 8 & 30 \\
Perforating branch (+) & 13 & 3 & 23 & 39 \\
\hline
\end{tabular}

Types I and III significantly different $\left(\mathrm{p}<0.01, \chi^{2}\right.$ test, odds ratio $=4.42$ ).
Table 3 Perforating branches from offending artery (PICA and AICA)

\begin{tabular}{lll}
\hline Offending artery & PICA & AICA \\
\hline Perforating branch (-) & 16 & 11 \\
Perforating branch (+) & 14 & 25 \\
\hline
\end{tabular}

$\mathrm{AICA}=$ anterior inferior cerebellar artery; $\mathrm{PICA}=$ posterior inferior cerebellar artery.

$p>0.05\left(\chi^{2}\right.$ test, odds ratio $\left.=2.6\right)$.

graphy, or 3-D CT angiography for some other condition to compare the radiological features of the vertebrobasilar configurations between patients with or without hemifacial spasm. As the control group did not have hemifacial spasm, it was difficult to categorise them into type I and type II patients. Therefore, the control group was divided into two classes: (1) the patients having a tortuous vertebrobasilar system; (2) the patients with a non-tortuous vertebrobasilar system. To maintain similarity and enable a statistical comparison between patients having hemifacial spasm and the control patients, the type I and type II patients in the hemifacial spasm group were also combined into the category of "tortuous type".

\section{Results}

The surgical treatment resulted in a complete relief in 55 patients $(80 \%)$ at the time of discharge, and in partial recovery in 14 patients $(20 \%)$. No patient was placed in the category of "no response". There was no operative mortality in the series. According to the vascular imaging, the 69 operated cases included 33 $(47.8 \%)$ patients of type I, five $(7.2 \%)$ of type II, and 31 of (45\%) type III. There was no significant difference in the sex, age, or the surgical result among the three types. The identity of the offending vessels in cases of each anatomical group is summarised in table 1 . The posterior inferior cerebellar artery (PICA)

Table 4 Comparison of vertebrobasilar configuration between hemifacial and control group

\begin{tabular}{lll}
\hline & Spasm group & Control group \\
\hline Tortuous type & 38 & 21 \\
non-tortuous type & 31 & 41
\end{tabular}

$\mathrm{p}<0.05\left(\chi^{2}\right.$ test, odds ratio $\left.=2.4\right)$. 

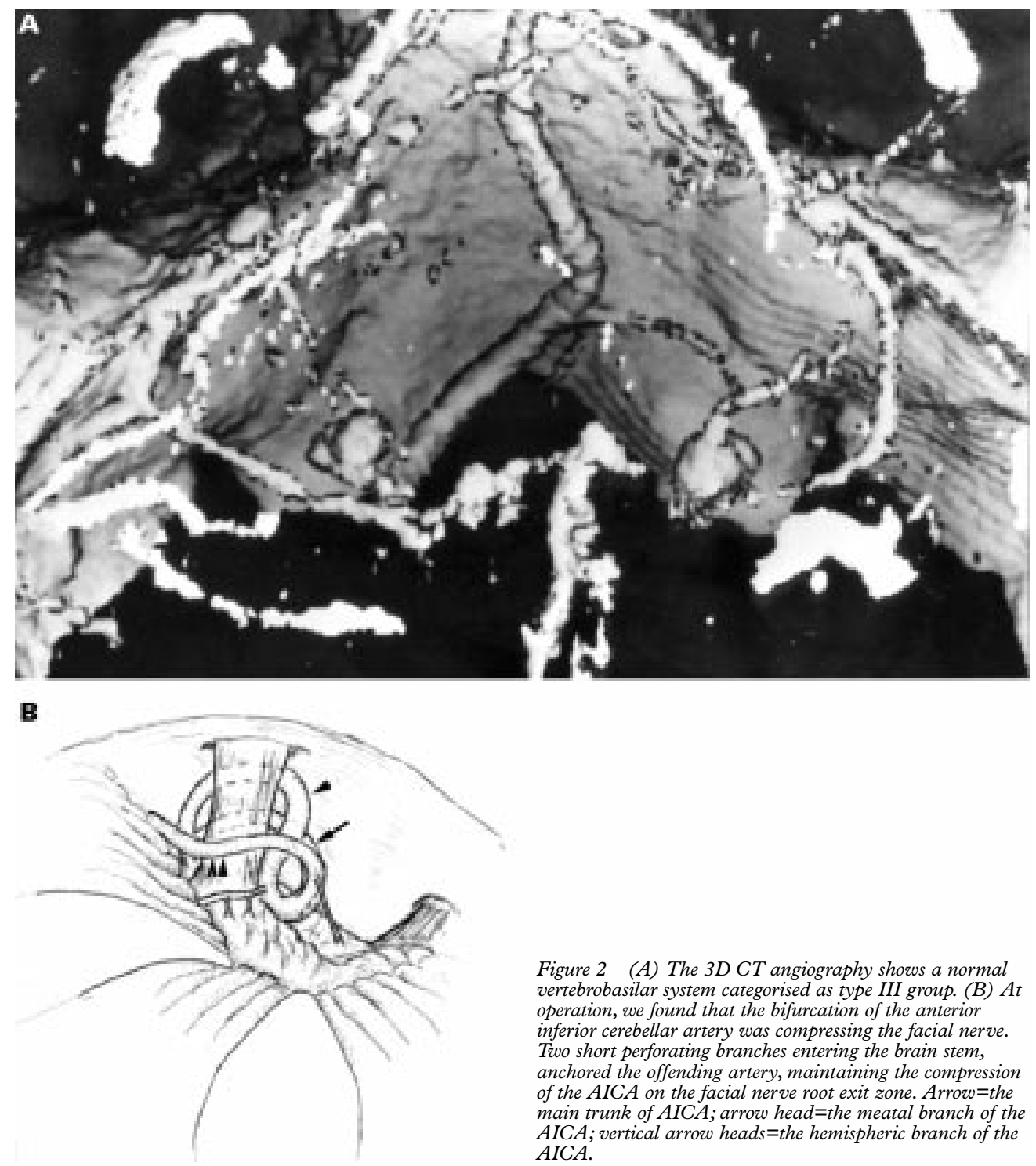

Figure 2 (A) The 3D CT angiography shows a normal vertebrobasilar system categorised as type III group. (B) At operation, we found that the bifurcation of the anterior inferior cerebellar artery was compressing the facial nerve. Two short perforating branches entering the brain stem, anchored the offending artery, maintaining the compression of the AICA on the facial nerve root exit zone. Arrow =the main trunk of AICA; arrow head=the meatal branch of the AICA; vertical arrow heads=the hemispheric branch of the $A I C A$.

was the most common offending vessel in type I $(73 \%)$, and the anterior inferior cerebellar artery (AICA), in both types II and III (100\% and $81 \%$ respectively). Table 2 summarises the number of the patients having perforating branches near the root entry zone of the VIIth nerve in each type. Twenty patients of the type I group showed no perforating branches whereas 12 patients showed perforating branches; and two of the type II group showed no perforating branches whereas three showed perforating branches; eight of the type III group had no perforating branches whereas 23 patients had them. The perforating branches tended to be more frequent in the AICA than in the PICA; however, the difference was not statistically significant (table 3 ). The control group consisted of 21 patients in the tortous type $(34 \%)$ and 41 in the non-tortous type $(64 \%)$. Table 4 shows that the difference in the ratio between the control group and the hemifacial spasm group reaches significance even though the mean age in the first group was 67.8 years compared with 50.6 in the second group.
ILLUSTRATIVE CASE

A 50 year old man presented with a right sided hemifacial spasm. The preoperative 3D CT angiography showed a rather straight vertebrobasilar configuration which could be categorised into type III (fig 2A). During surgery, we confirmed that the AICA was the offending artery and that it had two perforating branches penetrating the brain stem near the root exit zone. This significantly increased the difficulty in dissection to create an adequate space between the compressing vessel and the root entry zone (fig 2B).

\section{Discussion}

GENERAL ASPECTS

Hemifacial spasm is a syndrome that is spontaneous and gradual in onset and is characterised by intermittent twitching of the muscles of facial expression on one side of the face. Twitching typically begins about the eye and later spreads to involve the other muscles innervated by that facial nerve. ${ }^{3}$ In some patients, however, twitching begins in the buc- 
cal muscles and progresses over the face, a subtype described as an atypical hemifacial spasm. ${ }^{7}$ Our series included no cases of the atypical form.

Although several aetiologies have been proposed for hemifacial spasm, ${ }^{89}$ it is now widely accepted that it arises primarily from compression of the facial nerve at the root exit zone of the pons, usually by an adjacent artery. ${ }^{2} 3710$ The arteries involved in the typical hemifacial spasm include the PICA (22.9-59\%), the AICA (17-39\%), and vertebral artery (3.6-18\%). ${ }^{11-14}$ We found a similar incidence of the offending arteries in the present study.

ANATOMICAL EVIDENCE

Kondo et $a l^{15}$ delineated two characteristic configurations of the vertebrobasilar system in hemifacial spasm. In the first type, a tortuous vertebral artery on the affected side is often larger than the contralateral vertebral artery and gives rise to a redundant and dilated PICA. In the second type, an $S$ shaped basilar artery deviates from the midline and a tortuous AICA arising from it courses toward the internal auditory meatus on the affected side. In this category, usually the PICA is aplastic or hypoplastic. The first corresponds to type I and the second to the type II group of our series. We noted another characteristic configurationthat is, a normal vertebrobasilar system-and defined it as type III. This group comprised half of the total patients in our series. Among the offending vessels, the PICA was the most common compressing vessel in type I and the AICA in types II and III groups. Thus there was a significant difference between the type I and type III groups with regard to the offending vessels. On the other hand, Matsushima et $a l^{16}$ in a necropsy study examined 35 sides of the brain from 20 patients without hemifacial spasm and showed that in up to $70 \%$ of specimens, arterial attachment at the root entry zone was evident. They stated that the attachment was light and so did not manifest clinically as hemifacial spasm. Nearly half of our patients in the study as well as most of our patients in the control group had a non-tortuous vertebrobasilar artery and were categorised as type III patients. It is possible that there may be an attachment of the offending vessel to the VIIth nerve in our type III patients with hemifacial spasm similar to that seen in the series of normal patients by Matsushima et al, that became manifest. This group reported that the contacting vessels included the AICA in 24 patients, the PICA in two, and the vertebral artery in three In our series, the finding that the AICA is the most common offending vessel in the group III patients is, therefore, consistent with their findings. ${ }^{16}$

With regard to the perforating branches arising from the AICA and the PICA, Shrontz et $a l^{17}$ reported a unique study. They investigated the characteristics of the perforating branches in the posterior fossa using cadavaric dissection and ascertained the number of vessels, their origins, and their diameters to determine the best locations for anastomosis. According to this study, the PICA gives off only one or two perforating branches from the proximal $15 \mathrm{~mm}$ segment in the cisternal portion, but more branches arise from the distal cisternal portion. ${ }^{17}$ As the AICA extends from the basilar artery in a lateral and caudal direction, perforating branches are most concentrated at the distal portion of the bifurcation of the meatal and hemispheric branches where the compression point usually exists. ${ }^{5}{ }^{17}$ Matsushima et $a l^{16}$ also showed that in most of the patients, this part of the vessel is the portion causing the compression. These findings lead to the conclusion that in patients with hemifacial spasm with a normal vertebrobasilar system, some perforating branches from the AICA around the compressing point exist. However, the PICA is likely to give off perforating branches because it acts as an offending artery compressing the facial nerve root entry zone at the proximal cisternal portion in patients with a tortuous vertebral artery and in this portion of the PICA, usually the number of perforators is small. Our results were compatible with these conclusions (table 2). In the region of the root entry zone of the VIIth nerve, the AICA had more perforating branches than the PICA, but no significant difference was found (table 3 ).

In summary, the possibilities of encountering perforating branches while performing microvascular decompression for hemifacial spasm will increase as the vertebrobasilar configuration gets nearly normal.

We have presented the detailed findings from a 50 year old man with a right hemifacial spasm. The preoperative 3D CT angiography had shown a vertebrobasilar configuration curving to the unaffected side which could be categorised into the type III subgroup (fig 2A). At surgery, we confirmed that the AICA was the offending artery and that it had two perforating branches penetrating the brain stem near the root exit zone.This rendered the dissection to create adequate space between the compressing vessel and root entry zone extremely difficult (fig 2B).

The imaging information does not always predict the exact site of compression of the facial nerve. Some authors maintain that angiograms do not have a significant value in the management of hemifacial spasm. ${ }^{45}$ Since 1992, we have used a preoperative MRA or 3D CT angiography to exclude the existence of other vascular lesions. The purpose of our present study and our classification of the vertebrobasilar anatomy is not to identify the compressing artery but to attempt to correlate the configurations of the vertebrobasilar system with the perforating branches that are encountered during surgery for hemifacial spasm. Thus the radiological imaging will be helpful in identifying the patients in whom perforating branches will be encountered during microvascular decompression for hemifacial spasm, leading to a difficult dissection.

\section{Conclusion}

We retrospectively investigated 69 cases of hemifacial spasm, attempting to correlate the configuration of the vertebrobasilar system 
with the existence of short perforating branches near the root entry zone of the VII nerve. It is concluded that a symmetric, non-tortuous vertebrobasilar system is more likely to give rise to short perforating branches, which may cause difficulties during microvascular decompression.

1 Gardner WJ, Sava GA. Hemifacial spasm: a reversible pathophysiolosic state. $\mathcal{F}$ Neurosurg 1962;19:240-7.

2 . Thneta PI, Abbasy M Maroon JC, et al Etiology and Janneta PJ, Abbasy M, Maroon JC, et al. Etiology and
defenitive microsurgical treatment of hemifacial spasm. $\mathcal{F}$ defenitive microsurgical
Neurosurg 1977;47:321-8.

3 Wilkins R H. Hemifacial spasm: a review. Surg Neurol 1991; 36:251-77.

4 Sobel D, Norman D, Yorke CH, et al. Radiography of trigeminal neuralgia and hemifacial spasm. AfR Am $\mathcal{f}$ Roentgenol 1980;135:93-5.

5 Fukushima T. Posterior fossa microvascular decompression in the management of hemifacial spasm and trigeminal neuralgia. Neurol Surg 1988;10:1257-61.

6 Takeuchi T, Kasahara E. The use of Gore-tex vascular grafts in microvascular decompression for patiens with hemifacial spasm. Neurol Med Chir (Tokyo) 1993;33:111-13.

7 Janneta PJ. Hemifacial spasm. In: Samii M, Jannetta PJ, eds. The cranial nerves. Berlin: Springer-Verlag, 1981:484-93.

8 Adams CBT, Chir M. Microvascular compession: an alternative view and hypothesis. F Neurosurg 1989;57:1-12.
9 Aoki N, Nagao T. Resolution of hemifacial spasm after posterior fossa exploration without vascular decompression. terior fossa exploration with

10 Kim P, Fukushima T. Observations on synkinesis in patients with hemifacial spasm. I Neurosurg 1984;60:821-7.

11 Huang C I, Chen I H, Lee L S. Microvascular decompresion for hemifacial spasm: analyses of operative findings and results in 310 patients. Neurosurgery 1992;30:53-7.

12 Illingworth RD, Porter DG, Jakubowski J. Hemifacial spasm: a prospective long term follow up of 83 cases treated by microvascular decompression at two neurosurgichiatry 1996;60:72-7.

13 Loeser JD, Chen J. Hemifacial spasm: treatment by microsurgical facial nerve decompression. Neurosurgery 1983;13:141-6.

14 Piatt JH, Willkina RH. Treatment of tic douloureux and hemifacial spasm by posterior fossa exploration: therapeutic impliacation of various neurovascular relationships. Neurosurgery 1984;14:462-71.

15 Kondo A, Ishikawa I, Konishi T. The pathogenesis of hemifacial spasm: characteristic changes of vasculature in vertebral-basilar artery system. In: Samii M, Jannetta PJ, eds. The cranial nerves. Berlin: Springer-Verlag, 1981:494501.

16 Matsushima T, Inoue T, Fukui M. Arteries in contact with the cisternal portion of the facial nerve in autopsy cases: microsurgical anatomy for neurovascular decompression surgery of hemifacial spasm. Surg Neurol 1990;34:87-93.

17 Shrontz C, Dujovny M, Ausman J I, et al. Surgical anatomy of the arteries of the posterior fossa. I Neurosurg 1986;65:540-4. 